\title{
Clinical Trial Management Communication Documentation
}

National Cancer Institute

\section{Source}

National Cancer Institute. Clinical Trial Management Communication Documentation.

NCI Thesaurus. Code C115657.

Records detailing communications, agreements, significant discussions, or relevant information related to clinical trial management. 